\title{
Cord Blood Metabolome Is Highly Associated with Birth Weight, but Less Predictive for Later Weight Development
}

\author{
Christian Hellmuth $^{a} \quad$ Olaf Uhl $^{a} \quad$ Marie Standl $^{b}$ Hans Demmelmair ${ }^{a}$ \\ Joachim Heinrich $^{b, c} \quad$ Berthold Koletzko $^{a} \quad$ Elisabeth Thiering ${ }^{a, b}$ \\ a Division of Metabolic and Nutritional Medicine, Dr. von Hauner Children's Hospital, \\ University of Munich Medical Center, Ludwig-Maximilians-Universität München, Munich, \\ Germany; ${ }^{\text {b}}$ Institute of Epidemiology I, Helmholtz Zentrum München, German Research \\ Center for Environmental Health, Neuherberg, Germany; ${ }^{~}$ Institute and Outpatient Clinic \\ for Occupational, Social and Environmental Medicine, University of Munich Medical Center, \\ Ludwig-Maximilians-Universität München, Munich, Germany
}

\section{Key Words}

Cord blood · Metabolomics · Early programming $\cdot$ Childhood obesity

\begin{abstract}
Background/Aims: Fetal metabolism may be changed by the exposure to maternal factors, and the route to obesity may already set in utero. Cord blood metabolites might predict growth patterns and later obesity. We aimed to characterize associations of cord blood with birth weight, postnatal weight gain, and BMI in adolescence. Methods: Over 700 cord blood samples were collected from infants participating in the German birth cohort study LISAplus. Glycerophospholipid fatty acids (GPL-FA), polar lipids, non-esterified fatty acids (NEFA), and amino acids were analyzed with a targeted, liquid chromatography-tandem mass spectrometry based metabolomics platform. Cord blood metabolites were related to growth factors by linear regression models adjusted for confounding variables. Results: Cord blood metabolites were highly associated with birth weight. Lysophosphatidylcholines C16:1, C18:1, C20: 3, C18:2, C20:4, C14:0, C16:0, C18:3, GPL-FA C20:3n-9, and GPL-FA C22:5n-6 were positively related to birth weight, while higher cord blood concentrations of NEFA C22:6, NEFA C20:5, GPL-FA C18:3n-3, and PCe C38:0 were associated with lower birth weight. Postnatal weight gain and BMI z-scores in adolescents were not significantly associated with cord blood metabolites after adjustment for multiple testing. Conclusion: Potential long-term programming effects of the intrauterine environment and metabolism on later health cannot be predicted with profiling of the cord blood metabolome.

(C) 2017 The Author(s)

Published by S. Karger $\mathrm{GmbH}$, Freiburg
\end{abstract}

Prof. Dr. Berthold Koletzko

Division of Metabolic and Nutritional Medicine, Dr. von Hauner Children's Hospital

University of Munich Medical Center, Ludwig-Maximilians-Universität München

Lindwurmstraße 4, 80337 Munich, Germany

office.koletzko@ @ed.Imu.de 
Hellmuth et al.: Cord Blood Metabolome Is Highly Associated with Birth Weight, but Less Predictive for Later Weight Development

\section{Introduction}

The importance of the early life environment and metabolism on health and disease risk in later life is widely appreciated and has been referred to as 'early metabolic programming of adult health' $[1,2]$. Developmental plasticity during pre- and postnatal development allows the organism to adapt to environmental cues by cellular, metabolic, and other physiological changes. Hence different phenotypes can originate from the same genotype due to environmental stimuli via mechanisms such as epigenetic modifications [3]. For example, it has been proposed that intrauterine undernutrition can cause changes in endocrine response, body size, and composition as the fetus adapts to encounter the threat to survival $[4,5]$. Low birth weight and subsequent catch-up growth have both been associated with increased risk for later-life overweight $[6,7]$ and higher susceptibility to related diseases such as cardiovascular disease [8] and diabetes [9]. At the other end of the birth weight spectrum, later-life health consequences have also been observed: children of overweight mothers or children of women experiencing gestational diabetes are often born with high birth weights, and are subsequently at higher risk for overweight and diabetes later in life [9]. Thus, metabolic status at birth predefines later metabolic health. However, underlying genetic, epigenetic, and other mechanisms are still largely unknown. Metabolomic analyses have the potential to identify alterations in metabolic pathways and respective biomarkers [10]. In adults and children, associations between metabolite levels and overweight/obesity have been studied, and key molecules in amino acid (AA) and lipid metabolism have been identified [11]. For instance, branched-chain amino acids (BCAA), aromatic amino acids (AAA), non-esterified fatty acids (NEFA), acylcarnitines (AC), and phospholipids were identified as markers for obesity by targeted metabolomic approaches. In contrast, only very limited data is available relating cord blood metabolomic profiles to birth weight or later growth. Cord blood metabolites have been linked to low birth weight in infants with small for gestational age (SGA) [12] or intrauterine growth restriction (IUGR) [13, 14]. Only one study with a small sample size using an untargeted metabolomic approach linked cord blood metabolites to rapid postnatal weight gain [15]. Untargeted metabolomics offers the potential to determine a broad range of metabolites, but interpretation of the data may be limited by non-specificity for any given metabolite class and the lack of precise quantification [16]. Our aim was to depict associations not only to weight gain but also to birth weight and later BMI in a larger sample, with the goal to detect associations despite clinical heterogeneity regarding maternal BMI and dietary intake. We performed targeted quantitative metabolomic analysis including metabolites previously found to be altered in obesity. We aimed to evaluate possible early biomarkers for later obesity and to explore metabolic pathways related to early programming of obesity.

\section{Material and Methods}

\section{Study Population}

The 'Influence of life-style-factors on the development of the immune system and allergies in East and West Germany - Plus the influence of traffic emissions and genetics' study (LISAplus) enrolled 3,097 healthy, mature neonates with a birth weight at least 2,500 g from the cities of Munich, Leipzig, Wesel, and Bad Honnef between 1997 and 1999. LISAplus was designed as a population-based longitudinal birth cohort study with follow-up of children at the age of 6,12, and 18 months as well as 2, 4, 6, 10, and 15 years [17]. After birth, 4 $\mathrm{ml}$ of venous cord blood was collected and centrifuged at $1,400 \times \mathrm{g}$ for $10 \mathrm{~min}$. The serum was stored at $-20^{\circ} \mathrm{C}$ until IgE analysis and afterwards deep frozen at $-80^{\circ} \mathrm{C}$ until the time of metabolomics measurement [17].

A subsample of 753 children from the Munich and Bad Honnef study centers with available cord blood samples and anthropometric data was used for our analyses. The actual number of individuals differs between analyses depending on the number of missing values (e.g., in adjustment variables or metabolites); 
Hellmuth et al.: Cord Blood Metabolome Is Highly Associated with Birth Weight, but Less Predictive for Later Weight Development

therefore, this information is provided for each statistical model separately. Approval by the local Ethics Committees (Bavarian Board of Physicians, Board of Physicians of North-Rhine-Westphalia) and written consent from each participant's parents were obtained. The methods were carried out in accordance with the Declaration of Helsinki.

\section{Measurement of Metabolites}

Measurements were performed at the laboratory of the Division of Metabolic and Nutritional Medicine, Dr. von Hauner Children's Hospital, Ludwig-Maximilians-Universität München. Analysis of glycerophospholipid fatty acids (GPL-FA) was performed by transesterification of GPL-FA into methyl esters and their gas chromatographic separation, flame ionization detection (Agilent 7890A, Agilent Technologies, Mülheim an der Ruhr, Germany), and quantification as previously described [18]. Derivatization of fatty acids with sodium methoxide was followed by separation of fatty acid methyl esters by gas chromatography using a BPX70 (25 m × 0.22 mm; Phenomenex, Aschaffenburg, Germany) column. Polar lipids were analyzed with flow-injection analysis tandem mass spectrometry (MS) [19]. $10 \mu \mathrm{l}$ cord blood or standard solution was diluted with methanol, containing internal standards for different lipid groups and ammonium acetate. D3-carnitine C2, D3-carnitine C8, D3-carnitine C16, 13C6-D-glucose, (all Cambridge Isotope Laboratories, Tewksbury, MA, USA), acyl-lysophosphatidylcholines (LPCa) C13:0, and diacyl-phosphatidylcholines (PCa) C28:0 (both Avanti Polar Lipids, Alabaster, AL, USA) were used as internal standards. After centrifugation, supernatants were injected into a liquid chromatographic system (Agilent, Waldbronn, Germany) coupled to a triple quadrupole mass spectrometer (QTRAP4000, Sciex, Darmstadt, Germany) with an electrospray ionization source. The system was run in multiple reaction monitoring (MRM) mode in both positive and negative ionization mode. The analysis was performed on AC, diacyl- PCa, acyl-alkyl phosphatidylcholines (PCe), sphingomyelines (SM), LPCa, alkyl-lysophosphatidylcholines (LPCe) and sum of hexoses. The analytical technique applied here is not capable of determining the position of the double bonds and the distribution of carbon atoms between fatty acid side chains. AA analysis was performed as reported previously [20]. 10 $\mu \mathrm{l}$ cord blood or $10 \mu \mathrm{l}$ standard solution were diluted with the corresponding internal standard reagent. For internal standardization, a labelled AA standards set (set A; Cambridge Isotope Laboratories) was mixed with L-asparagine (15N2, 98\%; Cambridge Isotope Laboratories) and L-tryptophan (Indole-D5, 98\%; Cambridge Isotope Laboratories) and added to the precipitation reagent. Samples were prepared by derivatization to AA butyl ester, and determined by ion-pair liquid chromatography (HPLC 1100; Agilent) coupled to MS detection. MS detection was performed with an API 2000 triple quadrupole instrument (Sciex) with an atmospheric pressure chemical ionization source operating in positive ion ionization mode. The MS was run in MRM mode. Analysis of NEFA was performed as previously reported [21]. Briefly, $10 \mu \mathrm{l}$ cord blood or $10 \mu \mathrm{l}$ standard solution were mixed with $200 \mathrm{ml}$ isopropanol containing uniformly labelled palmitic acid (U-13C16, 98\%, Euriso-Top). After centrifugation the supernatant was used for HPLC-MS analysis. A triple quadrupole MS (4000 QTRAP; Sciex) operating in negative electrospray ionization mode was coupled to the HPLC system (Agilent 1200 SL series; Agilent) for identification of NEFA in MRM mode. NEFA are separated according to chain length and number of double bonds, but not according to position of double bonds.

The entire analytical process was controlled and post-processed by Analyst 1.6.1 and R software. GPL-FA, NEFA, and polar lipids are mentioned as CX:Y. In this nomenclature, $\mathrm{X}$ is the length of the carbon chain, $\mathrm{Y}$ is the number of double bonds, $\mathrm{OH}$ in the formula indicates that the molecule contains a hydroxyl group. ' $a$ ' indicates that the acyl chain is bound via an ester bond to the backbone, while 'e' indicates binding by an ether bond. All metabolites were identified by retention time and mass transition in tandem MS. We report all metabolite concentrations in $\mu \mathrm{mol} / \mathrm{l}$ cord blood. Seven ratios reflecting desaturation indexes for stearoyl-CoA desaturase-1 (SCD-1) were calculated by using the absolute concentrations of the selected metabolites [22].

\section{Outcome Measurement and Definition}

Birth weight as well as weight and height at 2 years of age were obtained from medical records. Weight and height at age 15 years were either measured at a clinical examination at the study center or obtained from questionnaires completed by the parents. Weight change after birth was defined as the difference of the weight at 6 months of age from the birth weight. To account for differences in age and sex, weight for length z-scores according to WHO growth standards at age 6 months and birth were used. BMI at ages 2 and 15 years were transformed to z-scores (zBMI) using age and sex-specific WHO growth references for school-aged children and adolescents [23]. 
Hellmuth et al.: Cord Blood Metabolome Is Highly Associated with Birth Weight, but Less Predictive for Later Weight Development

Statistical Analysis

To quantify the measurement accuracy, six plasma quality control samples were consistently measured twice along with the samples per batch. We calculated the coefficient of variation (CV) for each batch (intrabatch) and all of the batches (inter-batch). Regarding intra-batch precision, batches with a CV larger than $30 \%$ were excluded for single metabolite measurements, and complete metabolite measurements with an inter-batch $\mathrm{CV}>30 \%$ were excluded. Concentrations were log-transformed with base 2 , outliers exceeding median \pm 2.5 interquartile range were excluded, and metabolites with more than $50 \%$ missing values or samples with more than $50 \%$ of missing values were excluded from the analysis. To correct for potential batch effects in the liquid chromatography-tandem MS (LC-MS/MS) analysis, linear regression models were applied and the residuals were used for further statistical analysis.

The associations between metabolite concentrations in cord blood and birth weight were determined using linear regression models adjusted for study center, sex, gestational age, maternal smoking during the third trimester, maternal pre-pregnancy BMI, maternal weight gain during pregnancy, and maternal education (defined as low $=$ less than 10 years, medium $=10$ years and high $=$ more than 10 years of education). Sex-stratified analysis was performed for metabolites, which were significantly associated to birth weight. Cord blood metabolite levels of male and female newborns were compared by Mann-Whitey U test. To determine significant associations, Bonferroni correction for multiple testing was applied to all analyses yielding a significance level of $2.39 \times 10^{-4}$. Linear regression models for weight gain $\mathrm{z}$-scores included study center, sex, gestational age, and birth weight as adjustment variables, whereas the models for zBMI ( 2 and 15 years) were adjusted for study center, sex, and birth weight. Spearman correlation coefficients were calculated between effect estimates of metabolites for weight gain z-scores and zBMI at the age of 15 years. These linear regression models were also performed without adjusting for birth weight to check for over-adjustment. To determine the potential effect of maternal pre-pregnancy BMI, weight gain during pregnancy, and gestational diabetes, we additionally calculated linear regression models using cord blood metabolites as outcome for each maternal factor.

We related all cord blood metabolites that were either significantly associated with birth weight or that showed a false discovery rate (FDR) of less than $5 \%$ to later weight measures (weight gain z-score, zBMI at ages 2, and 15 years). Furthermore, Gaussian graphical models were applied to those metabolites to estimate the partial correlation structure. In this network, only correlations with a local FDR of $<5 \%$ for the partial correlation and a Pearson's correlation coefficient with $\mathrm{p}$ value $<0.10$ were displayed. All statistical analyses were performed using the statistical software R (3.1.3).

\section{Results}

Table 1 shows the characteristics of the study population. Some $18 \%$ of the mothers smoked at the beginning of pregnancy, whereas the percentage of smoking mothers decreased to $11 \%$ in the third trimester of the pregnancy. Birth weights were between 2,500 and 4,860 $\mathrm{g}(3,472 \pm 411 \mathrm{~g})$ with higher mean birth weights in boys $(3,542 \pm 403 \mathrm{~g})$ compared to girls $(3,394 \pm 406 \mathrm{~g} ; \mathrm{p}<0.001)$. Children of mothers who smoked during the third trimester of pregnancy showed lower birth weights (difference $159 \mathrm{~g} ; \mathrm{p}=0.001$ ). Compared to the WHO growth standards, infants in this study had higher birth weights and a lower postnatal weight gain which can be explained by the exclusion of infants with low birth weight or preterm birth. 209 metabolites were quantified with satisfactory precision in 753 cord blood samples of male and female newborns. Higher levels of cord AC C12:0 and C20:4n-6 were found in male newborns, while GPL-FA C16:0, C18:0, C18:1n-7, PCa C43:6, PCe C42:4, and SM C36:2 were significantly higher in female newborns (supplemental table 1, available at $h t t p: / /$ content.karger.com/ProdukteDB/produkte.asp?doi=453001). Most phospholipids tended to be higher in female, but AA were higher in cord plasma of male newborns (supplemental fig. 1, available at http://content.karger.com/ProdukteDB/produkte.asp?doi=453001).

\section{Birth Weight}

In the association analysis, significant differences related to birth weight were found for $2 / 37$ (4.2\%) of the measured NEFA, 3/22 (13.6\%) of the GPL-FA, 8/13 (61.5\%) of the LPC, 
Hellmuth et al.: Cord Blood Metabolome Is Highly Associated with Birth Weight, but Less Predictive for Later Weight Development

Table 1. Study population characteristics

\begin{tabular}{lll}
\hline & Study population & \\
\cline { 2 - 2 } & $\mathrm{n}(\%)$ or mean \pm SD & WHO z-score \\
\hline Study center & \\
$\quad$ Munich & $578(76.8)$ \\
$\quad$ Bad Honnef & $175(23.2)$ \\
Sex & \\
$\quad$ Male & $400(53.1)$ \\
Female & $353(46.9)$ \\
Gestational age, weeks & $40.0 \pm 1.2$ \\
Maternal pre-pregnancy BMI, kg/m ${ }^{2}$ & $22.5 \pm 4.0$ \\
Maternal weight gain during pregnancy, kg/month & $1.5 \pm 0.49$ \\
Maternal education & \\
$\quad$ Low & $75(10.1)$ \\
$\quad$ Medium & $237(31.8)$ \\
$\quad$ High & $434(58.2)$ \\
Maternal smoking during third trimester & \\
$\quad$ No & $641(89.3)$ \\
$\quad$ Yes & $77(10.7)$ \\
Birth weight, kg & $3.47 \pm 0.41$ \\
Early weight gain, kg/month & $0.7 \pm 0.13$ \\
BMI 2 years, kg/m ${ }^{2}$ & $16.1 \pm 1.4$ \\
Thin & $14(2.2)$ \\
Normal & $612(95.9)$ \\
Overweight/obese & $12(1.9)$ \\
BMI 15 years, kg/m ${ }^{2}$ & $20.5 \pm 3.1$ \\
$\quad$ Thin & $9(1.9)$ \\
$\quad$ Normal & $376(81.2)$ \\
$\quad$ Overweight/obese & $78(16.8)$ \\
\hline
\end{tabular}

Table 2. Associations of cord blood ratios for desaturation index of SCD-1 with birth weight and BMI at age 15 years $^{\mathrm{a}}$

\begin{tabular}{|c|c|c|c|c|c|c|}
\hline & \multicolumn{3}{|c|}{ Birth weight } & \multicolumn{3}{|c|}{ zBMI 15 years } \\
\hline & $\mathrm{N}$ & beta per SD & $\mathrm{p}$-value & $\mathrm{N}$ & beta per SD & p-value \\
\hline \multicolumn{7}{|l|}{ Nonesterified fatty acids } \\
\hline C16:1 / C16:0 & 661 & 8.8 & $5.32 \times 10^{-1}$ & 459 & 0.08 & $8.90 \times 10^{-2}$ \\
\hline $\mathrm{C} 17: 1$ / C17:0 & 661 & 26.8 & $5.55 \times 10^{-2}$ & 458 & 0.11 & $1.69 \times 10^{-2}$ \\
\hline C18:1 / C18:0 & 658 & 17.6 & $2.05 \times 10^{-1}$ & 458 & 0.13 & $8.90 \times 10^{-3}$ \\
\hline \multicolumn{7}{|l|}{ Glycerophospholipid fatty acids } \\
\hline C16:1n-7 / C16:0 & 662 & -7.6 & $5.92 \times 10^{-1}$ & 459 & -0.001 & $9.84 \times 10^{-1}$ \\
\hline C18:1n-9 / C18:0 & 661 & 12.2 & $3.94 \times 10^{-1}$ & 459 & -0.03 & $5.85 \times 10^{-1}$ \\
\hline \multicolumn{7}{|l|}{ Lysophosphatidylcholines } \\
\hline LPCa C16:1 / LPCa C16:0 & 660 & 89.0 & $1.35 \times 10^{-10}$ & 458 & -0.01 & $8.20 \times 10^{-1}$ \\
\hline LPCa C18:1 / LPCa C18:0 & 663 & 94.5 & $6.73 \times 10^{-11}$ & 460 & 0.03 & $5.98 \times 10^{-1}$ \\
\hline
\end{tabular}

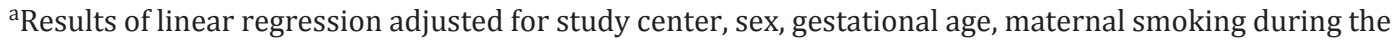
third trimester, maternal pre-pregnancy BMI, maternal weight gain during pregnancy and maternal education (birth weight) as well as for study center, sex and birth weight (zBMI 15 years). 
Hellmuth et al.: Cord Blood Metabolome Is Highly Associated with Birth Weight, but Less Predictive for Later Weight Development

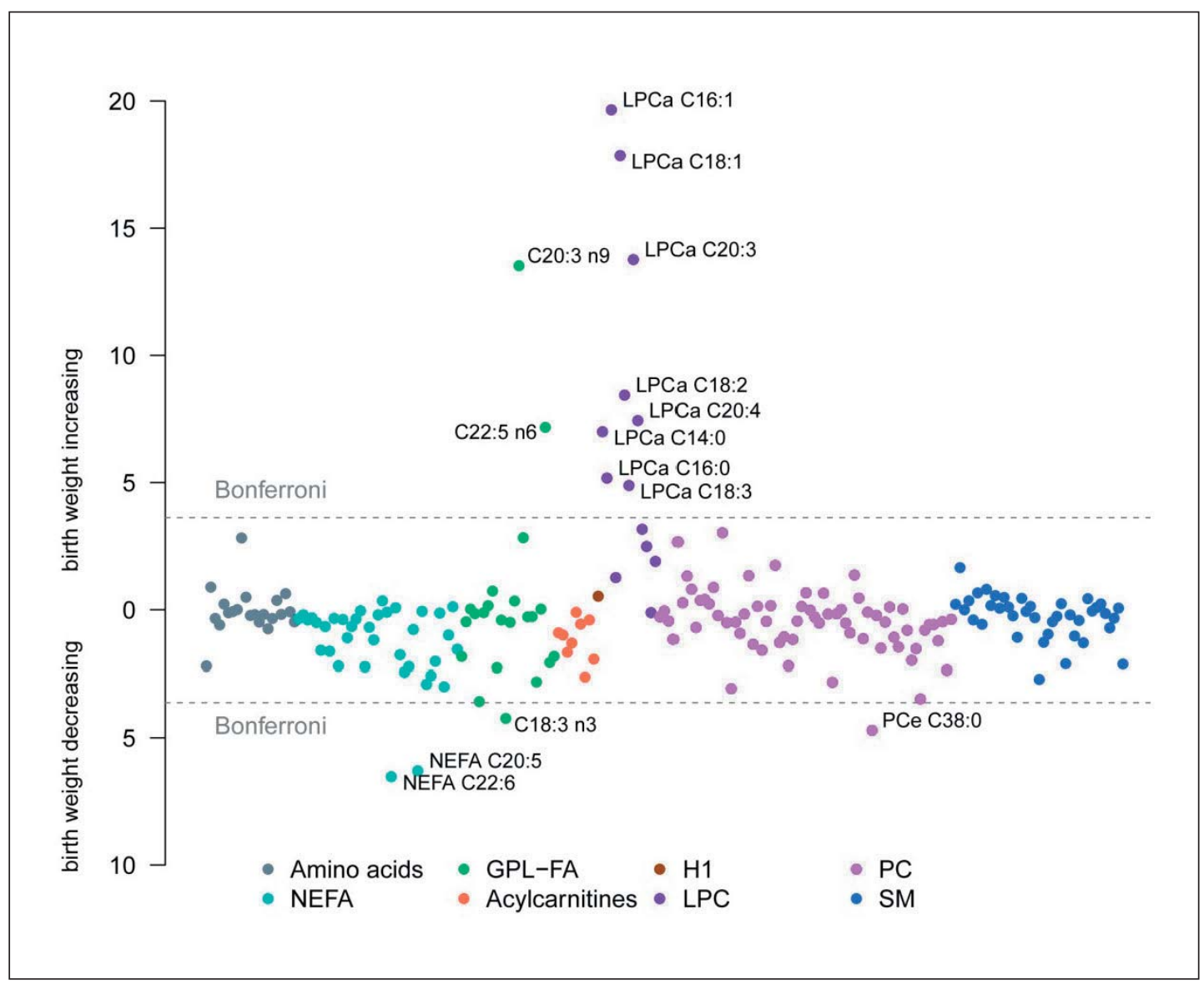

Fig. 1. Birth weight associations. Manhattan plot of the association between birth weight and metabolites adjusted for study center, sex, gestational age, maternal BMI, weight gain during pregnancy, smoking in the third trimester and education. Cx:y = An acyl group of chain length $\mathrm{x}$ and $\mathrm{y}$ double bounds; NEFA = nonesterified fatty acids; GPL-FA = glycerophospholipid fatty acids; LPC = lysophosphatidylcholines; PC = phosphatidylcholines; $\mathrm{SM}=$ sphingomyelins. Bonferroni significance level of $2.39 \times 10^{-4}$.

and 1/67 (1.5\%) of the PC after Bonferroni correction (fig. 1). For the 41 SM, 9 AC and 21 AA, no significant associations with birth weight were observed (supplemental table 2, available at http://content.karger.com/ProdukteDB/produkte.asp?doi=453001). Among the significant associations, all LPC had a positive relation to birth weight, while all NEFA and PCe C38:0 showed an inverse relationship to birth weight (fig. 1). Among the GPL-FA, C18:3n-3 were negatively associated with birth weight, while the association was positive for C20:3n-9 and C22:5n-6. The monounsaturated LPC C16:1 and C18:1 showed the strongest associations with birth weight, followed by LPC C20:3 and GPL-FA C20:3n-9. NEFA C20:5 and C22:6 showed the strongest negative association with birth weight. Ratios for the desaturation index of SCD-1 showed significant associations only for LPCs (table 2). The associations of NEFA C20:5 and C22:6 as well as LPC C18:2 and LPC C20:4 with birth weight were more pronounced in the female subgroup (fig. 2; supplemental table 3, available at $h t t p: / /$ content. karger.com/ProdukteDB/produkte.asp?doi=453001), but none of the birth weight-associated metabolites were related to the maternal factors pre-pregnancy BMI, maternal weight gain, or gestational diabetes (supplemental table 4, available at http://content.karger.com/ ProdukteDB/produkte.asp?doi=453001). 
Fig. 2. Sex stratified birth weight associations. Estimates of the association between birth weight and metabolites for male (blue) and female (red) subgroup adjusted for study center, sex, gestational age, maternal BMI, weight gain during pregnancy, smoking in the third trimester and education. NEFA = Nonesterified fatty acids; LPCa = lysophosphatidylcholines; PCe = acyl-alkyl-phosphatidylcholines. Bonferroni significance level of $2.39 \times 10^{-4}$.
Hellmuth et al.: Cord Blood Metabolome Is Highly Associated with Birth Weight, but Less Predictive for Later Weight Development

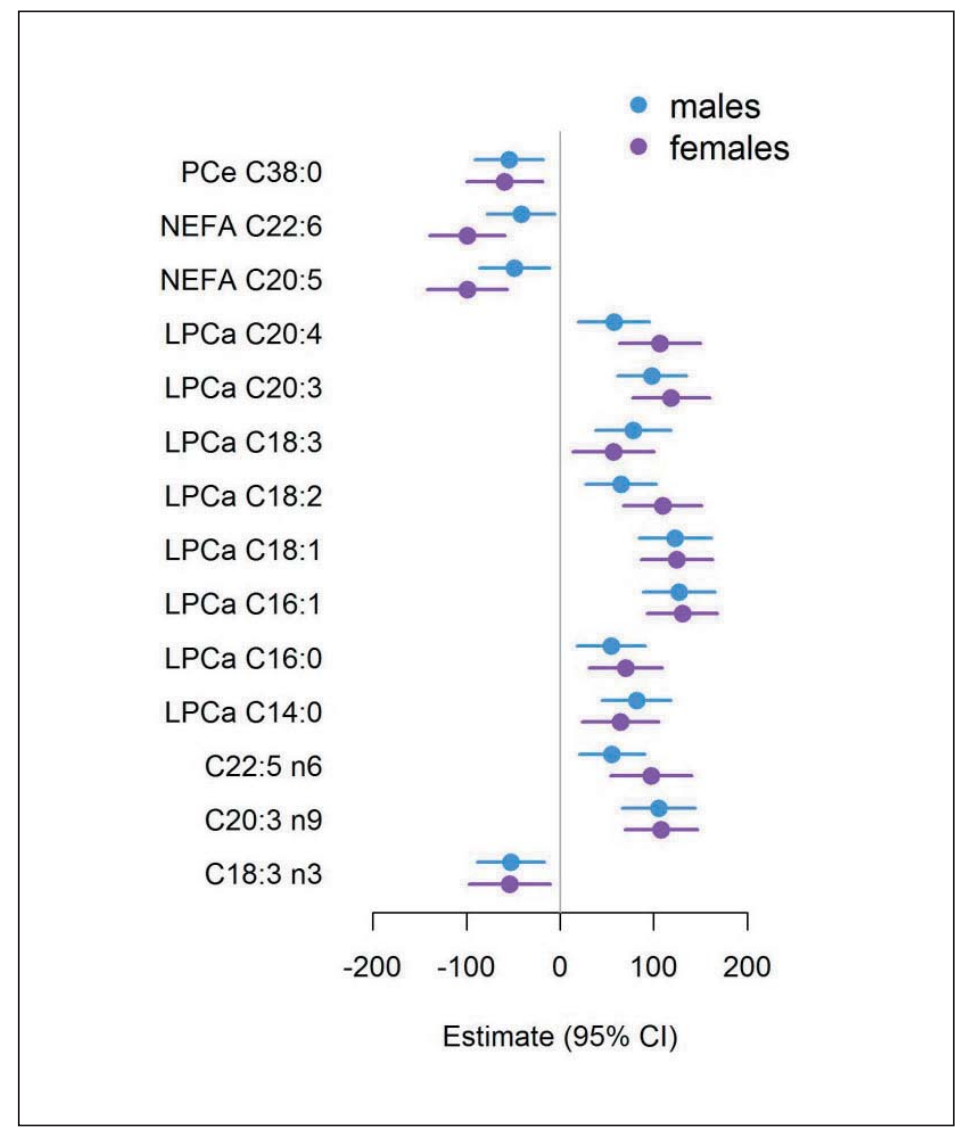

\section{Weight Gain / zBMI}

We observed a strong positive correlation (Spearman correlation $r=0.49, \mathrm{p}<0.001$ ) between estimates of metabolites for weight gain z-scores and zBMI at the age of 15 years (fig. 3). None of the associations of cord blood metabolites to later weight measures remained statistically significant after Bonferroni adjustment for multiple testing or FDR (supplemental table 2, available at $h t t p: /(c o n t e n t . k a r g e r . c o m / P r o d u k t e D B / p r o d u k t e . a s p ? d o i=453001)$. Also, linear regression models without adjusting for birth weight, which were performed to avoid over-adjustment, resulted in similar results (supplemental table 5; available at $h t t p: / /$ content. karger.com/ProdukteDB/produkte.asp?doi=453001). Cord blood metabolites that were associated with increased birth weight showed a tendency to be associated with lower postnatal weight gain z-scores and zBMI at ages 2 and 15 years (table 3). In contrast, cord blood metabolites with birth weight-lowering effects, had a tendency to be associated with increased postnatal weight gain z-scores and zBMI at age 15 years. For instance, higher NEFA levels were associated with lower birth weight followed by increased postnatal weight gain z-scores and consistently higher zBMI at the ages of 2 and 15 years (table 3). On the contrary, higher LPCa concentrations associated with higher birth weights were followed by lower postnatal weight gain z-scores and slightly lower zBMI at age 2 and at age 15 years. Interestingly, associations of cord blood metabolites with later zBMI showed similar effect sizes/magnitude of effect as with birth weight, although significance level were clearly stronger with birth weight (table 3; supplemental table 2, available at http://content.karger.com/ProdukteDB/produkte. asp?doi=453001). 
Hellmuth et al.: Cord Blood Metabolome Is Highly Associated with Birth Weight, but Less Predictive for Later Weight Development

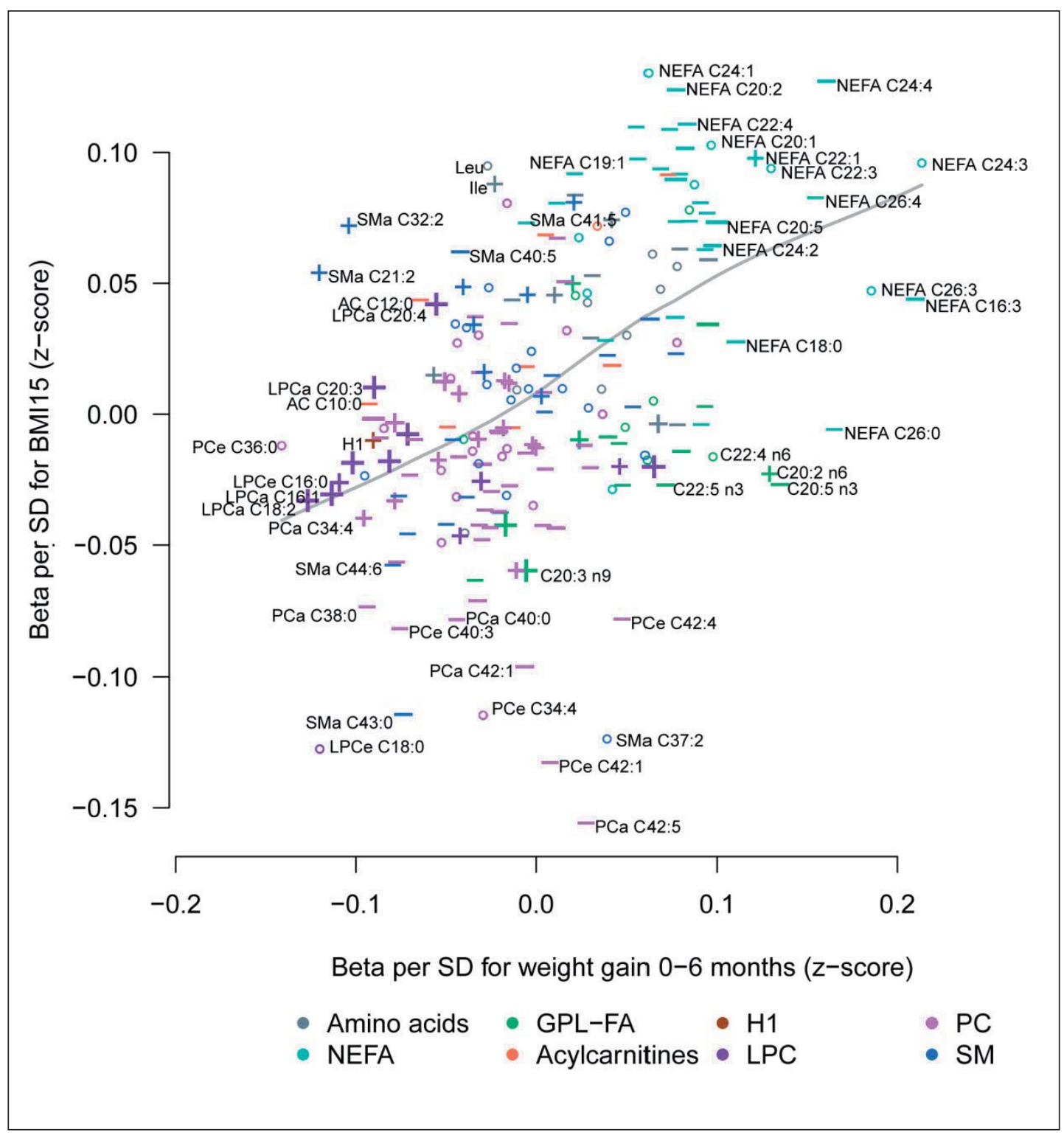

Fig. 3. Associations to weight gain and zBMI. Comparison of the associations between cord blood metabolites and weight gain during the first 6 months of life as well as zBMI at the age of 15 . + Birth weight-increasing metabolites (beta per SD > $10 \mathrm{~g}$ ), - birth weight-decreasing metabolites (beta per SD > $-10 \mathrm{~g}$ ), size of the symbols corresponds to the significance of the association with birth weight. Cx:y = An acyl group of chain length $\mathrm{x}$ and $\mathrm{y}$ double bounds; NEFA = nonesterified fatty acids; GPL-FA = glycerophospholipid fatty acids; LPC = lysophosphatidylcholines; $\mathrm{PC}=$ phosphatidylcholines; $\mathrm{SM}=$ sphingomyelins.

\section{Gaussian Graphical Models}

Figure 4 shows the network of partial correlations between the birth weight-associated metabolites. The highest positive partial correlations were observed between GPL-FA C22: 5n- 6 and GPL-FA C20:3n-9, GPL-FA C18:2n-6 and GPL-FA C20:3n-6, PCa C36:3 and GPL-FA C20:3n-6 as well as NEFA C22:4 and NEFA C22:5. Negative correlations were seen between GPL-FA C22:5n-3 and GPL-FA C20:3n-9, GPL-FA C22:5n-6 and GPL-FA C20:5n-3 as well as PCa C32:1 and LPCa C16:0. 
Hellmuth et al.: Cord Blood Metabolome Is Highly Associated with Birth Weight, but Less Predictive for Later Weight Development

Table 3. Cord blood metabolites showed a false discovery rate of less than $5 \%$ in association to birth weight, in relation to weight gain z-scores during first 6 months, BMI z-scores (zBMI) at ages 2 and 15 years

\begin{tabular}{|c|c|c|c|c|}
\hline & \multicolumn{4}{|c|}{ Association with } \\
\hline & birth weight & weight gain & zBMI 2 years & zBMI 15 years \\
\hline \multicolumn{5}{|l|}{ Amino acids } \\
\hline Ala & -- & ++ & + & ++ \\
\hline His & +++ & ++ & + & - \\
\hline \multicolumn{5}{|c|}{ Nonesterified fatty acids } \\
\hline C18:0 & -- & +++ & + & + \\
\hline $\mathrm{C} 20: 2$ & --- & ++ & + & +++ \\
\hline C22:6 & --- & ++ & - & ++ \\
\hline C16:3 & --- & +++ & + & + \\
\hline C18:4 & -- & ++ & + & + \\
\hline C20:5 & --- & ++ & + & ++ \\
\hline C22:4 & --- & ++ & -- & +++ \\
\hline $\mathrm{C} 22: 5$ & --- & ++ & - & +++ \\
\hline $\mathrm{C} 24: 2$ & --- & ++ & ++ & ++ \\
\hline C24:4 & --- & +++ & + & +++ \\
\hline \multicolumn{5}{|c|}{ Glycerophospholipid fatty acids } \\
\hline C17:0 & --- & ++ & + & - \\
\hline C18:2 n-6 & -- & + & + & - \\
\hline C18:3 n-3 & --- & ++ & - & + \\
\hline C20:3 n-9 & +++ & - & + & -- \\
\hline C20:3 n-6 & +++ & + & + & - \\
\hline C20:5 n-3 & --- & +++ & + & - \\
\hline $\mathrm{C} 22: 5 \mathrm{n}-6$ & +++ & - & + & - \\
\hline $\mathrm{C} 22: 5 \mathrm{n}-3$ & -- & ++ & - & - \\
\hline \multicolumn{5}{|c|}{ Acylcarnitines } \\
\hline $\mathrm{C} 2: 0$ & --- & + & + & + \\
\hline \multicolumn{5}{|c|}{ Lysophosphatidylcholines } \\
\hline LPCa C14:0 & +++ & -- & - & - \\
\hline LPCa C16:0 & +++ & -- & - & - \\
\hline LPCa C16:1 & +++ & --- & - & - \\
\hline LPCa C18:1 & +++ & --- & - & - \\
\hline LPCa C18:2 & +++ & --- & - & - \\
\hline LPCa C18:3 & +++ & ++ & + & - \\
\hline LPCa C20:3 & +++ & -- & - & + \\
\hline LPCa C20:4 & +++ & -- & - & + \\
\hline LPCa C22:6 & +++ & - & - & - \\
\hline LPCe C16:0 & +++ & --- & ++ & - \\
\hline
\end{tabular}

Table 3 continued on next page

\section{Discussion}

The intrauterine environment influences fetal development and infant growth in later life [24]. Different programming effects like maternal stress, smoking, and nutrition affect not only maternal but also fetal metabolism and may establish certain pathways like appetite regulation system, or gluconeogenesis in the fetus and the newborn, which have adverse effects in later life and result in childhood obesity and diabetes $[4,25]$. Thus, the newborn metabolome may not only be related to birth weight but may also predict later growth. In the presented study, we relate the cord blood metabolome of 750 newborns to their birth weight, 
Hellmuth et al.: Cord Blood Metabolome Is Highly Associated with Birth Weight, but Less Predictive for Later Weight Development

Table 3. Continued

\begin{tabular}{|c|c|c|c|c|}
\hline & \multicolumn{4}{|c|}{ Association with } \\
\hline & birth weight & weight gain & zBMI 2 years & zBMI 15 years \\
\hline \multicolumn{5}{|c|}{ Diacyl-phosphatidylcholine } \\
\hline PCa C32:1 & +++ & -- & - & - \\
\hline PCa C36:3 & +++ & -- & - & + \\
\hline PCa C36:5 & --- & - & + & - \\
\hline PCa C42:1 & --- & - & - & -- \\
\hline \multicolumn{5}{|c|}{ Acyl-akyl-phosphatidylcholine } \\
\hline PCe C34:3 & --- & + & - & - \\
\hline PCe C38:0 & --- & -- & - & - \\
\hline PCe C40:6 & --- & - & + & -- \\
\hline PCe C42:6 & --- & - & - & - \\
\hline \multicolumn{5}{|c|}{ Sphingomyelines } \\
\hline SM C40:5 & --- & - & + & ++ \\
\hline SM C42:4 & --- & ++ & + & + \\
\hline SM C43:0 & --- & -- & + & --- \\
\hline
\end{tabular}

--- Standardized effect estimate smaller than -0.10 ; -- effect estimate between -0.10 and -0.05 ; - effect estimate between -0.05 and 0 .

+++ Standardized effect estimate larger 0.10 ; ++ effect estimate between 0.05 and 0.10 ; + effect estimate between 0 and 0.05 .

Grey background: Significant after Bonferroni correction.

early weight gain, and zBMI at 2 and 15 years of age. Cord blood presents a mixture of maternal metabolism, placental transfer across the maternal-fetal axis and fetal metabolism. On the one hand, alterations in cord blood reflect differences in available nutrients for the fetus. For instance, placental nutrient transfer is reduced in baboon mothers with moderate nutrition restriction [26]. Additionally, changes in fetal metabolism are reflected in cord blood. Therefore, the strong association of several cord blood metabolites with birth weight is not unexpected. However, the predictive potential of these metabolites on later growth parameters is reduced. Postnatal factors, like early feeding practice, also influence infant development, and genetic programming is also strongly associated to growth [27]. For instance, Rzehak et al. [28] showed in the same cohort that infants who were fully breastfed, gained less weight in the first 3 months of life, and a slightly lower overweight risk was estimated for breastfed children. Meanwhile the metabolome might depend on various current environmental factors, such as diet, and thus the relation with phenotype parameters is higher in cross-sectional settings as displayed by the strong associations of cord blood metabolites to birth weight.

The strongest associations to birth weight were shown for NEFA and LPC, with different directions. Fatty acids are released from the placenta as NEFA into the fetal circulation [29]. Once taken up by the fetal liver, fatty acids can be incorporated predominantly in phospholipids and triacylglycerols. In contrast to NEFA, fetal phospholipids cannot be re-up-taken by the placenta [29]. Thus, cord NEFA present a mixture of maternal metabolism, placental function, and fetal lipid oxidation and storage, while phospholipids are a product of fatty acid supply to the fetus and fetal metabolism.

In adult metabolism, LPC in blood is derived from cleavage of PC by phospholipase A2, endothelial lipases [30] and lecithin-cholesterol acyltransferase [31]. The role of LPC in the fetal metabolism is unexplored, although their role in fatty acid transport from maternal 


\section{The European Journal of obesity}

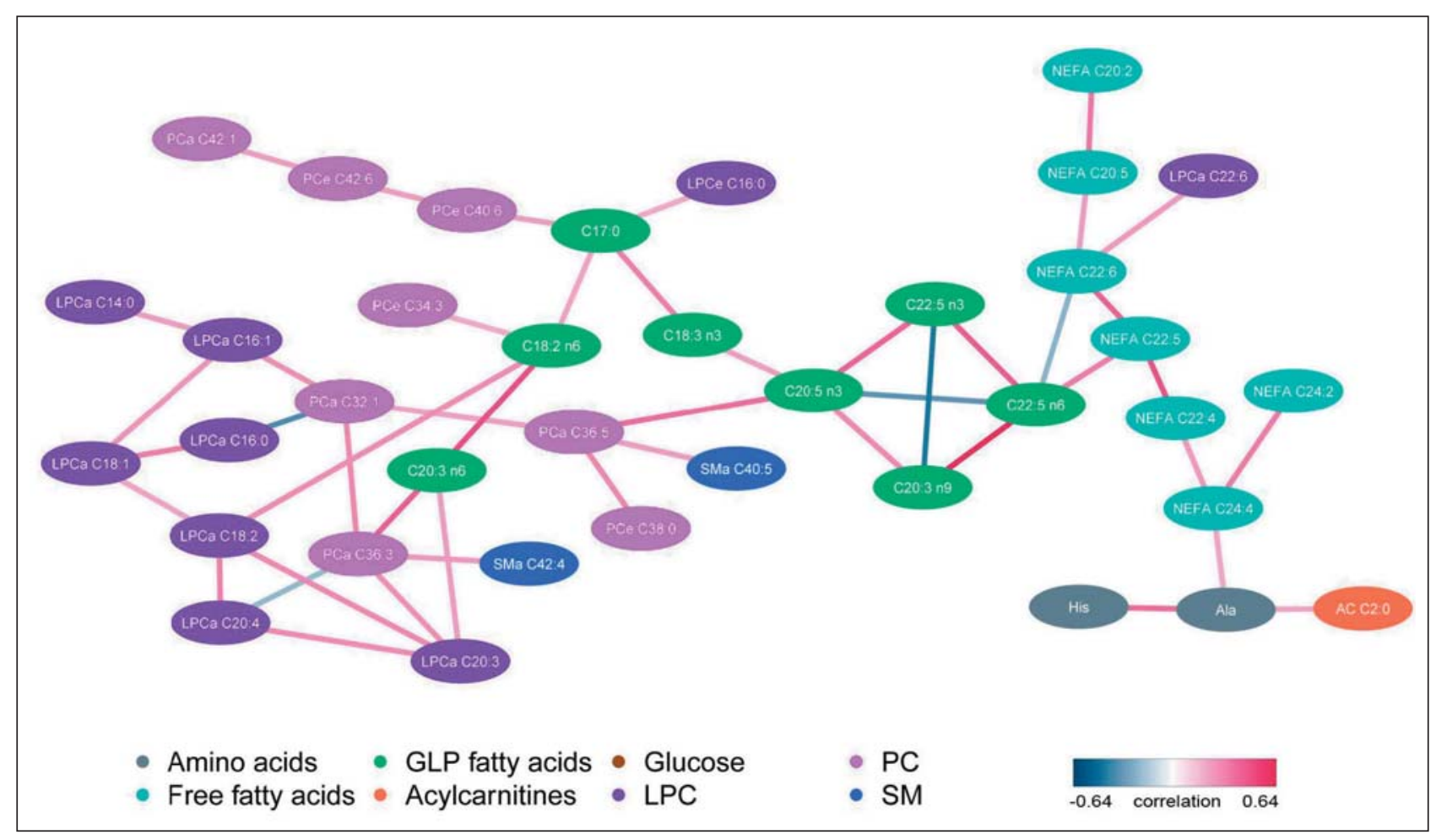

Fig. 4. Partial correlation network. Partial correlation network of cord blood metabolites associated with birth weight. Red lines display positive correlations, whereas blue lines stand for negative associations. Cx:y = An acyl group of chain length $\mathrm{x}$ and $\mathrm{y}$ double bounds; NEFA = nonesterified fatty acids; GPL fatty acids = glycerophospholipid fatty acids; LPC = lysophosphatidylcholines; PC = phosphatidylcholines; SM = sphingomyelins.

circulation into the placenta is of growing interest [32]. Our study demonstrated strong positive associations of cord blood LPC, in particular monounsaturated species, to birth weight, but no relation to the maternal factors pre-pregnancy BMI, gestational weight gain, and gestational diabetes. Although LPC are related to several inflammatory processes [30], their role in growth and obesity remains unclear. Higher levels of LPC species were reported in German obese men [33] and in obese monozygotic twins [34]. On the contrary, several LPC species were found to be lower in obese non-diabetic Australian adults [35] and in German obese adults [36]. Decreased levels in German obese children [37] were normalized by a 1-year lifestyle intervention [38]. Kim et al. [39] found higher (14:0, 18:0) and lower levels $(18: 1,18: 2,20: 2)$ of LPC in obese adults, which is in contrast to our study. Among the LPC, LPC 18:1 and 16:1 are the strongest predictors of birth weight. To depict the association of monounsaturated fatty acids (MUFA) to growth parameters, we also calculated ratios reflecting the desaturation index for SCD-1 [22]. The ratios of LPC 18:1 to 18:0 and 16:1 to 16:0 were positively associated to birth weight even after Bonferroni correction. Saturated fatty acid with a chain length of 16,17 and 18 carbon atoms are metabolized by SCD-1 to MUFA [40]. Further, SCD-1 is a potential key enzyme in the development of obesity-promoting lipogenesis rather than oxidation [41]. This increased lipogenesis results in larger fetal fat deposition and higher birth weight, both risk factors for later childhood obesity [42]. The third LPC which showed strong associations to birth weight was LPC 20:3. Since the position of the double bond is not determined by LC-MS/MS, this LPC is a mixture of the $n-3, n-6$, and n-9 isomer of 20:3. Although, 20:3n-9 in GPL showed a similar association with birth weight, 
Hellmuth et al.: Cord Blood Metabolome Is Highly Associated with Birth Weight, but Less Predictive for Later Weight Development

partial correlation (fig. 4) revealed a high correlation of LPC 20:3 to GPL-FA 20:3n-6, di-homo-gamma-linolenic acid (DGLA). DGLA is a known to be elevated in obese subjects of different age [43]. Maternal plasma PC with FA 20:3 were related to offspring adiposity [44], but placental PC species containing 20:3 were decreased in placenta of obese women [45].

In general, higher levels of single n-6 species (GPL FA C22:5n-6, LPC 20:3, LPC 20:4, LPC 18:2) were positively associated to birth weight, while the FA 18:3n-3 and the NEFA 20:5 and $22: 6$, presenting $n-3$ species [46], were negatively related to birth weight. A similar relation of n- 6 and n-3 to birth weight has been described previously, especially for C20:4n-6 [45, 47]. C20:4n-6 acts as precursor for growth-related prostaglandins [48]. In a recent study, lower maternal n-3 polyunsaturated fatty acid (PUFA) concentrations and higher $n-6$ PUFA concentrations during pregnancy were associated with higher body fat and abdominal fat in children of a large population-based, prospective cohort study [49]. High dietary n-6 fatty acid intakes and low n-3 intakes during pregnancy were reported as a risk indicator of later childhood obesity [50]. In rats, linoleic acid (18:2n-6) intake over generations increased adipose tissue mass [51], and its derivate 20:4n-6 is the main precursor of eicosanoids that can promote the differentiation of adipose precursor cells into adipocytes [52]. For the LISAplus cohort Munich, Standl et al. [53] showed an age-dependent effect of cord blood LC-PUFA on BMI up to 10 years of age.

In general, NEFA are elevated in obesity and positively associated to BMI and body weight [54], but association studies of cord blood NEFA to birth weight or fetal growth are missing. In this study, cord NEFA species showed a tendency to a negative association to birth weight. A possible explanation is a decreased lipolytic activity and enhanced NEFA uptake of the fetal adipose tissue. With greater fetal adipose tissue, a higher tissue NEFA uptake is expected, which would result in lower blood NEFA levels, along with higher body fat mass and birth weight. The positive associations of the LPC C18:2 and C20:4 as well as the negative association of the NEFA C20:5 and C22:6 to birth weight were more pronounced in female newborns who have a higher body fat mass than male neonates, although the tendency was similar in boys and girls. Thus, the changes in n- 3 and n- 6 species may have different effects in girls and boys, while alteration in MUFA metabolism is not related to sex. Furthermore, we found a trend to higher phospholipids, but lower AA levels in cord blood of female newborns. This is in contrast to recent findings by Ruoppolo et al. [55] who reported higher AA and lower AC levels in blood spots of female newborns compared to male ones. Additionally, the associations of AA to birth weight were more pronounced in male subjects, while Ruoppolo et al. [55] found AA important to describe female body weight in cord blood. In our study, we found no association of AA to birth weight. This is in contrast to previous studies, since AA were found to be associated to IUGR and SGA in newborns $[12,14]$. However, children with low birth weight were excluded from the study, and thus we could not confirm these findings in our study. A further explanation might be the influence of placental and maternal metabolism. Alteration in AA metabolism during pregnancy [56] and different transfer of AA across the placenta [57] strongly affect cord blood. However, peripheral blood, which is collected in blood spots from heel peek, might be less affected. The influence of maternal sources on the metabolome enhances the variation in AA levels and may also explain the missing association of AA to birth weight.

In contrast to the associations at birth, the metabolomic profile was not associated to weight gain and later BMI. The absolute value of estimates of associations between weight gain and obesity were comparable to the estimates of 'birth weight associations'. Thus, the effect size was similar but the significance strongly lower for the later growth parameters, probably by interfering effects of infant feeding, later dietary intake, and other environment factors on later growth, which additional increased the variation in growth development. This missing association is in contrast to the only other study determining the association of cord 
Hellmuth et al.: Cord Blood Metabolome Is Highly Associated with Birth Weight, but Less Predictive for Later Weight Development

blood metabolites and postnatal weight gain done by Isganaitis et al. [15] who found lower tryptophan and methyl donor metabolites in the cord blood of infants with rapid postnatal weight gain.

The presented low association of cord blood metabolome to later obesity in our cohort may be a result of the small number of obese children in our cohort. Thus, the prevalence of obesity may be too low to predict the later obesity risk by the cord metabolome. Additionally, infants with low birth weight were excluded due to the study design. Given the fact that low birth weight is a predictor for childhood obesity, we could not study the metabolic relation of IUGR to later obesity. To avoid possible over-adjustment, we also performed models without adjustment for birth weight, but we did not detect a change in the associations.

Other reasons for the missing predictive power of the cord metabolome for later growth can be due to the experiment conditions, e.g., contaminations of cord blood by maternal blood during collection as seen for IgE [58]. Only few studies analyze the effect of storage time on the blood metabolome. Most of these studies focus on higher temperature $\left(-20^{\circ} \mathrm{C}\right.$, dry ice), different kind of samples (urine), or lower periods of observation time compared to the presented study [59-62]. In general, these studies found less influence of storage time at -20 or $-80^{\circ} \mathrm{C}$ or freezing cycles on the metabolome. Further, one study found no influence of storage temperature or length of long-term storage in biobanks up to 17 years on the metabolomic profiles [63]. Additionally, pre-storage parameters, like time until freezing, affect the metabolites. Anton et al. [64] showed that in particular AA and LPC increase and PCa decrease due hydrolysis by longer storage at room temperature before processing. Given the very strong associations between birth weight and certain cord metabolites (e.g., LPC), we assume that the quality of the cord blood samples and the measurement did not in principle affect the presented study, but other metabolites, in particular AA, which were not associated to birth weight and later growth parameters, may have been influenced by the experimental conditions. Thus, we assume that storage conditions are not responsible for missing predictive power of the cord metabolome to later growth, and we conclude that the measured metabolites within these studies are less predictive. Further studies should thus focus on other potential targets like hormones [65], the epigenome [66], or other metabolites [15], which have the potential to predict later obesity risk. However, the large sample size of our study cohort allowed us to depict the associations of cord blood metabolome to fetal and infant growth. We could demonstrate that cord blood metabolites are highly associated with birth weight, but have less potential to predict patterns of later weight and growth. Thus, possible programming effects of the intrauterine environment and metabolism on later growth outcomes are not reflected by cord blood. These programming effects may rather occur in fetal/newborn organs like the liver or in the (epi-)genome, or they may be linked to postnatal growth patterns that compensate for deviations of birth weight. Association analysis of birth weight revealed a strong association to cord LPC, a potential marker for fetal liver metabolism. SCD-1 activity and seems to be enhanced with increasing birth weight and may result in increased fat storage in the newborn.

\section{Acknowledgements}

The authors thank all the families for their participation in the LISAplus study. We thank Stefanie Winterstetter and Stefan Stromer (Division of Metabolic and Nutritional Medicine, Dr. von Hauner Children's Hospital, Ludwigs-Maximilians-Universität München) who prepared the plasma samples for GC and LC-MS/ MS analysis.

Furthermore, we thank all members of the LISAplus Study Group for their excellent work.

The LISAplus Study group consists of the following: Helmholtz Zentrum München, German Research Center for Environmental Health, Institute of Epidemiology I, Munich (Heinrich J, Schnappinger M, Brüske I, 
Hellmuth et al.: Cord Blood Metabolome Is Highly Associated with Birth Weight, but Less Predictive for Later Weight Development

Sußmann M, Lohr W, Schulz H, Zeller C, Standl M); Department of Pediatrics, Municipal Hospital 'St. Georg', Leipzig (Borte M, Gnodtke E); Marien Hospital Wesel, Department of Pediatrics, Wesel (von Berg A, Berdel D, Stiers G, Maas B); Pediatric Practice, Bad Honnef (Schaaf B); Helmholtz Center of Environmental Research - UFZ, Department of Environmental Immunology/Core Facility Studies, Leipzig (Lehmann I, Bauer M, Röder S, Schilde M, Nowak M, Herberth G , Müller J, Hain A); Technical University Munich, Department of Pediatrics, Munich (Hoffmann U, Paschke M, Marra S); Clinical Research Group Molecular Dermatology, Department of Dermatology and Allergy, Technical University Munich (Ollert M).

\section{Funding}

The LISAplus study was supported by the Federal Ministry for Education, Science, Research and Technology, the Helmholtz Center for Environmental Research Leipzig, and the Helmholtz Zentrum Munich. Follow-up examinations were additionally supported by the Leibniz Research Institute for Environmental Medicine, Düsseldorf and the Commission of the European Communities through the 7th Framework Programme MeDALL project (No. 261357). The metabolomics analyses were financially supported by the Commission of the European Communities 7th Framework Programme, contract FP7-289346-EARLY NUTRITION, and European Research Council Advanced Grant ERC-2012-AdG - No. 322605 META-GROWTH. This paper does not necessarily reflect the views of the Commission and in no way anticipates the future policy in this area. Additional support by the Kompetenznetz Adipositas (Competence Network Obesity), Federal Ministry of Education and Research (FKZ 01GI1121A) is gratefully acknowledged.

\section{Disclosure Statement}

The authors have declared that have no conflict of interest.

\section{References}

1 Barker DJ, Osmond C: Infant mortality, childhood nutrition, and ischaemic heart disease in England and Wales. Lancet 1986;1:1077-1081.

2 Koletzko B, Brands B, Chourdakis M, Cramer S, Grote V, Hellmuth C, Kirchberg F, Prell C, Rzehak P, Uhl O, Weber M: The power of programming and the earlynutrition project: opportunities for health promotion by nutrition during the first thousand days of life and beyond. Ann Nutr Metab 2014;64:187-196.

3 Sookoian S, Gianotti TF, Burgueno AL, Pirola CJ: Fetal metabolic programming and epigenetic modifications: a systems biology approach. Pediatr Res 2013;73:531-542.

4 Hales CN, Barker DJ: The thrifty phenotype hypothesis. Br Med Bull 2001;60:5-20.

5 Okada T, Takahashi S, Nagano N, Yoshikawa K, Usukura Y, Hosono S: Early postnatal alteration of body composition in preterm and small-for-gestational-age infants: implications of catch-up fat. Pediatr Res 2015;77: 136-142.

6 Ong KK, Emmett PM, Noble S, Ness A, Dunger DB, Team AS: Dietary energy intake at the age of 4 months predicts postnatal weight gain and childhood body mass index. Pediatrics 2006;117:e503-508.

7 Singhal A, Lucas A: Early origins of cardiovascular disease: is there a unifying hypothesis? Lancet 2004;363: 1642-1645.

8 Huxley R, Owen CG, Whincup PH, Cook DG, Rich-Edwards J, Smith GD, Collins R: Is birth weight a risk factor for ischemic heart disease in later life? Am J Clin Nutr 2007;85:1244-1250.

9 Harder T, Rodekamp E, Schellong K, Dudenhausen JW, Plagemann A: Birth weight and subsequent risk of type 2 diabetes: a meta-analysis. Am J Epidemiol 2007;165:849-857.

10 Coughlin SS: Toward a road map for global -omics: a primer on -omic technologies. Am J Epidemiol 2014;180: 1188-1195.

11 Rauschert S, Uhl O, Koletzko B, Hellmuth C: Metabolomic biomarkers for obesity in humans: a short review. Ann Nutr Metab 2014;64:314-324.

12 Ivorra C, Garcia-Vicent C, Chaves FJ, Monleon D, Morales JM, Lurbe E: Metabolomic profiling in blood from umbilical cords of low birth weight newborns. J Transl Med 2012;10:142.

13 Alexandre-Gouabau MC, Courant F, Moyon T, Kuster A, Le Gall G, Tea I, Antignac JP, Darmaun D: Maternal and cord blood LC-HRMS metabolomics reveal alterations in energy and polyamine metabolism, and oxidative stress in very-low birth weight infants. J Proteome Res 2013;12:2764-2778.

14 Favretto D, Cosmi E, Ragazzi E, Visentin S, Tucci M, Fais P, Cecchetto G, Zanardo V, Viel G, Ferrara SD: Cord blood metabolomic profiling in intrauterine growth restriction. Anal Bioanal Chem 2012;402:1109-1121. 
Hellmuth et al.: Cord Blood Metabolome Is Highly Associated with Birth Weight, but Less Predictive for Later Weight Development

15 Isganaitis E, Rifas-Shiman SL, Oken E, Dreyfuss JM, Gall W, Gillman MW, Patti ME: Associations of cord blood metabolites with early childhood obesity risk. Int J Obes (Lond) 2015;39:1041-1048.

16 Gibbons H, O'Gorman A, Brennan L: Metabolomics as a tool in nutritional research. Curr Opin Lipidol 2015; 26:30-34.

17 Heinrich J, Bolte G, Holscher B, Douwes J, Lehmann I, Fahlbusch B, Bischof W, Weiss M, Borte M, Wichmann HE, Group LS: Allergens and endotoxin on mothers' mattresses and total immunoglobulin E in cord blood of neonates. Eur Respir J 2002;20:617-623.

18 Glaser C, Demmelmair H, Koletzko B: High-throughput analysis of total plasma fatty acid composition with direct in situ transesterification. PLoS One 20109;5:e12045.

19 Rauschert S, Uhl O, Koletzko B, Kirchberg F, Mori TA, Huang RC, Beilin LJ, Hellmuth C, Oddy WH: Lipidomics reveals associations of phospholipids with obesity and insulin resistance in young adults. J Clin Endocrinol Metab 2016;101:871-879.

20 Harder U, Koletzko B, Peissner W: Quantification of 22 plasma amino acids combining derivatization and ion-pair LC-MS/MS. J Chromatogr B Analyt Technol Biomed Life Sci 2011;879:495-504.

21 Hellmuth C, Weber M, Koletzko B, Peissner W: Nonesterified fatty acid determination for functional lipidomics: comprehensive ultrahigh performance liquid chromatography-tandem mass spectrometry quantitation, qualification, and parameter prediction. Anal Chem 2012;84:1483-1490.

22 Paton CM, Ntambi JM: Biochemical and physiological function of stearoyl-CoA desaturase. Am J Physiol Endocrinol Metab 2009;297:E28-37.

23 de Onis M, Onyango AW, Borghi E, Siyam A, Nishida C, Siekmann J: Development of a WHO growth reference for school-aged children and adolescents. Bull World Health Organ 2007;85:660-667.

24 Koletzko B, Chourdakis M, Grote V, Hellmuth C, Prell C, Rzehak P, Uhl O, Weber M: Regulation of early human growth: impact on long-term health. Ann Nutr Metab 2014;65:101-109.

25 Kabaran S, Besler H: Do fatty acids affect fetal programming? JJ Health Popul Nutr 2015;33:14.

26 Pantham P, Rosario FJ, Nijland M, Cheung A, Nathanielsz PW, Powell TL, Galan HL, Li C, Jansson T: Reduced placental amino acid transport in response to maternal nutrient restriction in the baboon. Am J Physiol Regul Integr Comp Physiol 2015;309:R740-746.

27 Tounian P: Programming towards childhood obesity. Ann Nutr Metab 2011;58(suppl 2):30-41.

28 Rzehak P, Sausenthaler S, Koletzko S, Bauer CP, Schaaf B, von Berg A, Berdel D, Borte M, Herbarth O, Kramer U, Fenske N, Wichmann HE, Heinrich J: Period-specific growth, overweight and modification by breastfeeding in the GINI and LISA birth cohorts up to age 6 years. Eur J Epidemiol 2009;24:449-467.

29 Haggarty P: Fatty acid supply to the human fetus. Annu Rev Nutr 2010;30:237-255.

30 Sevastou I, Kaffe E, Mouratis MA, Aidinis V: Lysoglycerophospholipids in chronic inflammatory disorders: The PLA(2)/LPC and ATX/LPA axes. Biochim Biophys Acta 2013;1831:42-60.

31 Jonas A: Lecithin cholesterol acyltransferase. Biochim Biophys Acta 2000;1529:245-256.

32 Prieto-Sanchez MT, Ruiz-Palacios M, Blanco-Carnero JE, Pagan A, Hellmuth C, Uhl O, Peissner W, Ruiz-Alcaraz AJ, Parrilla JJ, Koletzko B, Larque E: Placental MFSD2A transporter is related to decreased DHA in cord blood of women with treated gestational diabetes. Clin Nutr 2016;36:513-521.

33 Graessler J, Schwudke D, Schwarz PE, Herzog R, Shevchenko A, Bornstein SR: Top-down lipidomics reveals ether lipid deficiency in blood plasma of hypertensive patients. PLoS One 2009;4:e6261.

34 Pietilainen KH, Sysi-Aho M, Rissanen A, Seppanen-Laakso T, Yki-Jarvinen H, Kaprio J, Oresic M: Acquired obesity is associated with changes in the serum lipidomic profile independent of genetic effects - a monozygotic twin study. PLoS One 2007;2:e218.

35 Barber MN, Risis S, Yang C, Meikle PJ, Staples M, Febbraio MA, Bruce CR: Plasma lysophosphatidylcholine levels are reduced in obesity and type 2 diabetes. PLoS One 2012;7:e41456.

36 Heimerl S, Fischer M, Baessler A, Liebisch G, Sigruener A, Wallner S, Schmitz G: Alterations of plasma lysophosphatidylcholine species in obesity and weight loss. PLoS One 2014;9:e111348.

37 Wahl S, Yu Z, Kleber M, Singmann P, Holzapfel C, He Y, Mittelstrass K, Polonikov A, Prehn C, Romisch-Margl W, Adamski J, Suhre K, Grallert H, Illig T, Wang-Sattler R, Reinehr T: Childhood obesity is associated with changes in the serum metabolite profile. Obes Facts 2012;5:660-670.

38 Reinehr T, Wolters B, Knop C, Lass N, Hellmuth C, Harder U, Peissner W, Wahl S, Grallert H, Adamski J, Illig T, Prehn C, Yu Z, Wang-Sattler R, Koletzko B: Changes in the serum metabolite profile in obese children with weight loss. Eur J Nutr 2015;54:173-181.

39 Kim JY, Park JY, Kim OY, Ham BM, Kim HJ, Kwon DY, Jang Y, Lee JH: Metabolic profiling of plasma in overweight/obese and lean men using ultra performance liquid chromatography and Q-TOF mass spectrometry (UPLC-Q-TOF MS). J Proteome Res 2010;9:4368-4375.

40 Brett D, Howling D, Morris LJ, James AT: Specificity of the fatty acid desaturases. The conversion of saturated to monoenoic acids. Arch Biochem Biophys 1971;143:535-547.

41 Hulver MW, Berggren JR, Carper MJ, Miyazaki M, Ntambi JM, Hoffman EP, Thyfault JP, Stevens R, Dohm GL, Houmard JA, Muoio DM: Elevated stearoyl-CoA desaturase-1 expression in skeletal muscle contributes to abnormal fatty acid partitioning in obese humans. Cell Metab 2005;2:251-261.

42 Koletzko B, Beyer J, Brands B, Demmelmair H, Grote V, Haile G, Gruszfeld D, Rzehak P, Socha P, Weber M: Early influences of nutrition on postnatal growth. Nestle Nutr Inst Workshop Ser 2013;71:11-27.

43 Fekete K, Gyorei E, Lohner S, Verduci E, Agostoni C, Decsi T: Long-chain polyunsaturated fatty acid status in obesity: a systematic review and meta-analysis. Obes Rev 2015;16:488-497. 
Hellmuth et al.: Cord Blood Metabolome Is Highly Associated with Birth Weight, but Less Predictive for Later Weight Development

44 de Vries PS, Gielen M, Rizopoulos D, Rump P, Godschalk R, Hornstra G, Zeegers MP: Association between polyunsaturated fatty acid concentrations in maternal plasma phospholipids during pregnancy and offspring adiposity at age 7:The MEFAB cohort. Prostaglandins Leukot Essent Fatty Acids 2014;91:81-85.

45 Uhl O, Demmelmair H, Segura MT, Florido J, Rueda R, Campoy C, Koletzko B: Effects of obesity and gestational diabetes mellitus on placental phospholipids. Diabetes Res Clin Pract 2015;109:364-371.

46 Hodson L, Skeaff CM, Fielding BA: Fatty acid composition of adipose tissue and blood in humans and its use as a biomarker of dietary intake. Prog Lipid Res 2008;47:348-380.

47 Crawford M: Placental delivery of arachidonic and docosahexaenoic acids: Implications for the lipid nutrition of preterm infants. Am J Clin Nutr 2000;71(1 suppl):275S-284S.

48 Markworth JF, Cameron-Smith D: Arachidonic acid supplementation enhances in vitro skeletal muscle cell growth via a cox-2-dependent pathway. Am J Physiol Cell Physiol 2013;304:C56-67.

49 Vidakovic AJ, Gishti O, Voortman T, Felix JF, Williams MA, Hofman A, Demmelmair H, Koletzko B, Tiemeier H, Jaddoe VW, Gaillard R: Maternal plasma PUFA concentrations during pregnancy and childhood adiposity: the Generation R Study. Am J Clin Nutr 2016;103:1017-1025.

50 Innis SM: Fatty acids and early human development. Early Hum Dev 2007;83:761-766.

51 Massiera F, Barbry P, Guesnet P, Joly A, Luquet S, Moreilhon-Brest C, Mohsen-Kanson T, Amri EZ, Ailhaud G: A western-like fat diet is sufficient to induce a gradual enhancement in fat mass over generations. J Lipid Res 2010;51:2352-2361.

52 Ailhaud G, Guesnet P, Cunnane SC: An emerging risk factor for obesity: does disequilibrium of polyunsaturated fatty acid metabolism contribute to excessive adipose tissue development? Br J Nutr 2008;100:461-470.

53 Standl M, Thiering E, Demmelmair H, Koletzko B, Heinrich J: Age-dependent effects of cord blood long-chain PUFA composition on BMI during the first 10 years of life. Br J Nutr 2014;111:2024-2031.

54 Klop B, Elte JW, Cabezas MC: Dyslipidemia in obesity: mechanisms and potential targets. Nutrients 2013;5: 1218-1240.

55 Ruoppolo M, Scolamiero E, Caterino M, Mirisola V, Franconi F, Campesi I: Female and male human babies have distinct blood metabolomic patterns. Mol Biosyst 2015;11:2483-2492.

56 Lindsay KL, Hellmuth C, Uhl O, Buss C, Wadhwa PD, Koletzko B, Entringer S: Longitudinal metabolomic profiling of amino acids and lipids across healthy pregnancy. PLoS One 2015;10:e0145794.

57 Battaglia FC, Regnault TR: Placental transport and metabolism of amino acids. Placenta 2001;22:145-161.

58 Bonnelykke K, Pipper CB, Bisgaard H: Transfer of maternal IgE can be a common cause of increased IgE levels in cord blood. J Allergy Clin Immunol 2010;126:657-663.

59 Dunn WB, Broadhurst D, Ellis DI, Brown M, Halsall A, O’Hagan S, Spasic I, Tseng A, Kell DB: A GC-TOF-MS study of the stability of serum and urine metabolomes during the UK biobank sample collection and preparation protocols. Int J Epidemiol 2008;37(suppl 1):i23-30.

60 Gika HG, Theodoridis GA, Wilson ID: Liquid chromatography and ultra-performance liquid chromatographymass spectrometry fingerprinting of human urine: sample stability under different handling and storage conditions for metabonomics studies. J Chromatogr A 2008;1189:314-322.

61 Rist MJ, Muhle-Goll C, Gorling B, Bub A, Heissler S, Watzl B, Luy B: Influence of freezing and storage procedure on human urine samples in NMR-based metabolomics. Metabolites 2013;3:243-258.

62 Zivkovic AM, Wiest MM, Nguyen UT, Davis R, Watkins SM, German JB: Effects of sample handling and storage on quantitative lipid analysis in human serum. Metabolomics 2009;5:507-516.

63 Hebels DG, Georgiadis P, Keun HC, Athersuch TJ, Vineis P, Vermeulen R, Portengen L, Bergdahl IA, Hallmans G, Palli D, Bendinelli B, Krogh V, Tumino R, Sacerdote C, Panico S, Kleinjans JC, de Kok TM, Smith MT, Kyrtopoulos SA; EnviroGenomarkers Project Consortium: Performance in omics analyses of blood samples in long-term storage: opportunities for the exploitation of existing biobanks in environmental health research. Environ Health Perspect 2013;121:480-487.

64 Anton G, Wilson R, Yu ZH, Prehn C, Zukunft S, Adamski J, Heier M, Meisinger C, Romisch-Margl W, Wang-Sattler R, Hveem K, Wolfenbuttel B, Peters A, Kastenmuller G, Waldenberger M: Pre-analytical sample quality: metabolite ratios as an intrinsic marker for prolonged room temperature exposure of serum samples. PLoS One 2015;10:e0121495.

65 Brandt S, Moss A, Lennerz B, Koenig W, Weyermann M, Rothenbacher D, Brenner H, Wabitsch M: Plasma insulin levels in childhood are related to maternal factors - results of the Ulm Birth Cohort Study. Pediatr Diabetes 2014;15:453-463.

66 Soubry A, Schildkraut JM, Murtha A, Wang F, Huang Z, Bernal A, Kurtzberg J, Jirtle RL, Murphy SK, Hoyo C: Paternal obesity is associated with IGF2 hypomethylation in newborns: results from a Newborn Epigenetics Study (NEST) cohort. BMC Med 2013;11:29. 